\title{
JORNALEROS INMIGRANTES EN LA AGRICULTURA MURCIANA: REFLEXIONES SOBRE CRISIS, INMIGRACIÓN Y EMPLEO AGRÍCOLA
}

\section{IMMIGRANT WORKERS IN AGRICULTURE IN MURCIA: REFLECTIONS ON CRISIS, IMMIGRATION AND AGRICULTURAL LABOR}

María Elena Gadea, Carlos de Castro, Andrés Pedreño y Natalia Moraes*

Resumen: En las últimas décadas, España ha basado su crecimiento económico en sectores intensivos en mano de obra, caracterizados por condiciones laborales precarias, donde los trabajadores inmigrantes han jugado un papel fundamental ante la escasez de mano de obra autóctona. El artículo reflexiona sobre los vínculos entre uno de estos sectores, la agricultura intensiva de exportación, y el empleo de trabajadores extranjeros, con especial atención a la segregación por género y etnia de este mercado de trabajo, las condiciones laborales de los jornaleros inmigrantes y al impacto de la actual crisis económica sobre el empleo en este sector.

Palabras clave: migraciones laborales, trabajo agrícola, agricultura intensiva, crisis económica

* En este artículo se presentan los resultados del proyecto de investigación «Sostenibilidad social de los nuevos enclaves productivos agrícolas: España y México», dirigido por Andrés Pedreño Cánovas y financiado por el Ministerio de Ciencia e Innovación (2012-2014, CSO2011-28511). 
Abstract: The Spanish economic growth in recent decades has been based on labor-intensive economic sectors (construction industry, tourism-related services and export agriculture). In these sectors, characterized by precarious labor conditions, migrant workers have played a key role as a labor force available and underpaid. This article reflects on the links between the economic model of intensive export agriculture and the use of foreign labor, with special attention to the working conditions of migrant workers and the impact of the current economic crisis on the employment in this sector.

Keywords: labor migration, agricultural workers, intensive agriculture, economic crisis

Los emigrantes bullían en los caminos, y en sus ojos se retrataba su hambre, y sus privaciones estaban marcadas en sus ojos. No tenían argumentos ni sistemas, nada, sino un número y sus necesidades. Cuando había trabajo para un hombre, diez hombres luchaban por conseguirlo..., y su arma era ofrecer sus servicios por menos dinero (...). Y esto era conveniente, porque bajaban los salarios y se mantenía el precio. Los grandes propietarios estaban contentos y repartían más prospectos para atraer a más gente. Los salarios bajaban y los precios se mantenían al mismo nivel.

John Steinbeck, Las uvas de la ira

\section{INTRODUCCIÓN}

Como otras regiones del Sur de Europa, el estado español ha basado su crecimiento económico reciente en actividades intensivas en mano de obra y dependientes de mercados externos, como la construcción, el turismo o la agricultura intensiva, con unas relaciones laborales caracterizadas por la eventualidad, la precariedad y los bajos salarios ${ }^{1}$. En este modelo de desarrollo, los flujos migratorios

1 Leborgne y Lipietz (1994) definen esta opción de desarrollo como estrategias defensivas de tipo neotayloriano (escasa innovación, trabajo temporal de bajos salarios, mano de obra inmigrante sin apenas derechos de ciudadanía), a 
internacionales se orientaron hacia las regiones donde la modernización económica transfería hacia otros sectores las bolsas de trabajadores eventuales autóctonos, generando problemas de escasez en los sectores más tradicionales y segmentando los mercados de trabajo ${ }^{2}$. De este modo, el reclutamiento de trabajadores inmigrantes se convirtió en la condición de posibilidad para la reproducción de un capitalismo meridional que perpetuaba la eventualidad y que hacía de la contención de los costes laborales uno de sus elementos básicos de competitividad (de Castro, Gadea y Pedreño, 2014). Desde finales de los años 90 hasta mediados de la década pasada, el abundante empleo permitió a estos trabajadores inmigrantes insertarse en la sociedad española, aunque en posiciones vulnerables. La crisis ha supuesto un punto de inflexión en estos procesos, mostrando tanto la insostenibilidad del modelo de desarrollo como la debilidad de las situaciones de inserción de los migrantes.

La construcción ha sido, sin duda, la apuesta fundamental de este modelo. Menos atención se ha puesto en otros sectores, como la agricultura intensiva de exportación, que también ha tenido y tiene un protagonismo fundamental en las estrategias de desarrollo de la vertiente mediterránea española y que ha jugado un papel básico en la inserción socio-laboral de los trabajadores inmigrantes, dado su carácter de nicho laboral etnificado y de puerta de entrada al mercado de trabajo. En este sentido, la producción intensiva de hortalizas y frutas en fresco para los mercados internacionales se presenta como un escenario privilegiado para preguntarse por los impactos

las que oponen las estrategias ofensivas de tipo kalmariano (implicación negociada, altos salarios) desarrolladas por las regiones ricas del Norte de Europa. Para estos autores, las regiones que garantizasen estrategias ofensivas-kalmarianas se erigirían en «regiones ganadoras» y las que, por el contrario, adoptasen estrategias de tipo defensivo y neo-taylorista se configurarían como «regiones perdedoras».

2 En los años setenta Doeringer y Piore (1985) nos advertían de que los mercados de trabajo no son homogéneos sino que están segmentados en un mercado primario (empleos cualificados, bien remunerados y con promoción) y en un mercado secundario (empleos inestables, remuneración modesta y sin promoción). Además, señalaron que esta segmentación del mercado de trabajo era también una segmentación social. Los estadounidenses blancos tendían a ocupar los puestos del mercado primario y la población negra y latina tendía a ocupar los del mercado secundario. Desde entonces, esta pauta de segmentación se ha ido repitiendo en diversos contextos variando la calidad del empleo y los colectivos segmentados. 
de la crisis y, en particular, sobre sus efectos para los trabajadores inmigrantes, especialmente en un contexto como el actual en el que desde la política económica se apuesta por el fortalecimiento de los sectores de exportación como salida a la crisis ${ }^{3}$.

El artículo reflexiona sobre los vínculos entre el modelo de agricultura intensiva de exportación y el empleo de mano de obra extranjera, con especial atención al impacto de la actual crisis económica sobre este sector. Nos aproximaremos a estos fenómenos a partir de una doble mirada. La primera, centrada en el conjunto del territorio español, analiza el papel de las migraciones laborales en el desarrollo de la agricultura de exportación y la constitución de este sector como nicho laboral segmentado por género y etnia. La segunda mirada se dirige a un territorio concreto, la Región de Murcia, una zona emblemática de agricultura intensiva de exportación que constituye un buen «laboratorio» para analizar tanto los vínculos entre reestructuraciones económicas y migraciones internacionales, como la inserción de los migrantes en los enclaves productivos agrícolas ${ }^{4}$.

3 Las exportaciones agroalimentarias españolas alcanzaron en 2013 un valor total de 38.000 millones de euros y representan el 16,2\% del total de exportaciones nacionales; entre los subsectores más relevantes destacan las frutas y las hortalizas, que se exportan por un valor de 7.000 y 4.800 millones de euros al año respectivamente (MAGRAMA, 2014). Precisamente este subsector, el hortofrutícola, es uno de los más dinámicos de la agricultura española y tiene un «carácter estratégico para la economía española», ya que «ocupa el primer puesto dentro de las exportaciones agrícolas y el quinto del total de las exportaciones». España es el primer país europeo en exportación de frutas y hortalizas y el segundo en producción, después de Italia (SEPE, 2013:15-16).

4 La base empírica de este trabajo procede de diversas investigaciones que, desde hace más de una década, hemos venido desarrollando en la Región de Murcia sobre el empleo en la agricultura y las migraciones internacionales, utilizando fundamentalmente técnicas cualitativas (entrevistas, grupos de discusión, historias de vida y observación). En lo que respecta al impacto de la crisis sobre las condiciones laborales en el sector agrícola, el trabajo de campo ha consistido en la realización de entrevistas a trabajadores de campo y almacén, empresarios agrícolas, responsables sindicales e intermediarios laborales entre noviembre de 2012 y septiembre de 2014, en el marco del proyecto Sostenibilidad social de los nuevos enclaves productivos agrícolas: España y México (CSO2011-28511). 


\section{AGRICULTURA DE EXPORTACIÓN Y MIGRACIONES DEL TRABAJO EN EL MODELO DE DESARROLLO ESPAÑOL.}

El importante desarrollo de la agricultura intensiva en España no puede entenderse sin el trabajo de los jornaleros extranjeros. La llegada de inmigrantes a las zonas agrícolas españolas está ligada al incremento de las necesidades de trabajo asalariado en la agricultura, como consecuencia de su intensificación y de la pérdida de peso de la ayuda familiar, en un contexto en el que los jornaleros autóctonos estaban abandonando el trabajo agrícola para moverse hacia otros sectores económicos, como la construcción y los servicios. El primer flujo migratorio vinculado a la agricultura se produce, a mediados de los 70 y principios de los 80 , hacia las explotaciones familiares de hortalizas, flores y fruta de varias comarcas catalanas. A finales de la década de los 80 y principios de los 90 encontramos un segundo flujo, esta vez hacia el litoral mediterráneo, en particular, hacia las zonas de regadío e invernaderos de Murcia y Almería (Giménez, 1992; Avellá y García, 1995, Gozálvez y López, 1999). Estas primeras migraciones estuvieron protagonizadas por varones jóvenes, de origen magrebí y subsahariano, que se movían por el territorio español siguiendo las cosechas o se instalaban en las zonas de agricultura intensiva del mediterráneo, en particular cerca de grandes ciudades que ampliaran las posibilidades de inserción laboral y residencial. La especialización productiva, el predominio de la pequeña propiedad familiar y la estacionalidad del trabajo hacía que estos jornaleros debieran moverse continuamente dentro de las provincias en que estaban asentados y también de unas provincias a otras (Gozálvez y López, 1999:218).

Con el desarrollo del modelo de agricultura intensiva, en las zonas donde se ha dado una mayor desestacionalización de la producción y se ha favorecido la sedentarización de los jornaleros, como Murcia y Almería, encontramos importantes procesos de asentamiento. La necesidad continua de trabajadores, el aumento y la estabilización del empleo, las oportunidades laborales en sectores no agrícolas y los procesos de regularización y de reagrupación familiar, contribuyen a fijar espacialmente a estos nuevos jornaleros (Gadea, Ramírez y Sánchez, 2014), aunque las migraciones circulares o pendulares siguen estando presentes y juegan un papel fundamental en el suministro de fuerza de trabajo. Otros enclaves agrícolas, en 
cambio, siguen teniendo una demanda de trabajo marcada por una alta estacionalidad. Es el caso de Huelva, Jaén o Albacete, donde los periodos de trabajo en la fresa, la aceituna o la vid, están muy acotados temporalmente. Esto hace que un volumen importante de jornaleros deba moverse hacia ellos, durante un periodo de tiempo limitado, para participar en diferentes tareas agrícolas. Para regular estos flujos, se estableció en 1997 el Convenio Marco de Colaboración para la Ordenación de las Migraciones Interiores en las Diversas Campañas Agrícolas de Empleo Temporal, que dos años más tarde serviría de base para la puesta en marcha de los programas de contratación en origen de trabajadores extranjeros para diversas campañas agrícolas, particularmente en Huelva y Lleida (Achón, 2011; Torres et al., 2014).

\subsection{La conformación de un espacio laboral segmentado por etnia y género.}

La evolución de los mercados laborales agrícolas en España ha venido marcada, en gran medida, por la búsqueda de estrategias de fijación y de movilización continua de trabajadores, con el fin de cubrir las necesidades de trabajo barato y disponible en una agroindustria que ha tratado de contener los costes laborales para producir en condiciones de competitividad. La política de extranjería y la gestión empresarial del trabajo ha cumplido una importante función en estos procesos. Mediante el Régimen General y el Contingente, la normativa española ha favorecido la concentración de la población inmigrante en determinadas ocupaciones.

Las estadísticas de afiliados a la Seguridad Social muestran cómo los trabajadores extranjeros se encuentran sobrerrepresentados en el Sistema Especial Agrario y el Sistema Especial de Empleados de Hogar ${ }^{5}$, donde en 2013 se concentraban el 12\% y el 14\%, respectivamente, de los extranjeros afiliados. En el caso de los trabajadores

5 A partir del 1 de enero de 2012, los trabajadores por cuenta ajena del Régimen Especial Agrario pasan a integrarse en el Régimen General, como un Sistema Especial para Trabajadores por Cuenta Ajena Agrarios, de acuerdo a lo establecido en la Ley 28/2011 de 22 de septiembre. Desde esa misma fecha, los trabajadores del Régimen Especial de Empleados de Hogar pasan a integrarse en el Régimen General, como un Sistema Especial de Empleados de Hogar, de acuerdo a lo establecido en la Ley 27/2011, de 1 de agosto. 
españoles, el porcentaje para esos sectores era del 4,6\% en el sector agrario y del $2,6 \%$ en el servicio doméstico (tabla 1 ).

\section{TABLA 1}

\section{AFILIADOS A LA SEGURIDAD SOCIAL SEGÚN RÉGIMEN (\% SOBRE EL TOTAL POR NACIONALIDAD)}

\begin{tabular}{|c|c|c|c|c|c|c|c|c|}
\hline & \multicolumn{6}{|c|}{ Régimen General } & \multirow{2}{*}{\multicolumn{2}{|c|}{ Otros regímenes }} \\
\hline & \multicolumn{2}{|c|}{ General } & \multicolumn{2}{|c|}{ Especial Agrario } & \multicolumn{2}{|c|}{$\begin{array}{c}\text { Especial Empleados } \\
\text { de Hogar }\end{array}$} & & \\
\hline & Españoles & Extranjeros & Españoles & Extranjeros & Españoles & Extranjeros & Españoles & Extranjeros \\
\hline 2005 & 75,6 & 68,5 & 5,9 & 9,8 & 1,6 & 12,0 & 16,9 & 9,7 \\
\hline 2006 & 76,2 & 70,4 & 5,4 & 8,5 & 1,8 & 12,2 & 16,7 & 8,9 \\
\hline 2007 & 76,8 & 74,0 & 5,1 & 7,6 & 1,4 & 8,1 & 16,7 & 10,3 \\
\hline 2008 & 76,4 & 71,5 & 3,9 & 9,0 & 1,5 & 8,0 & 18,2 & 11,6 \\
\hline 2009 & 75,6 & 67,1 & 4,5 & 12,5 & 1,6 & 9,2 & 18,4 & 11,2 \\
\hline 2010 & 75,5 & 65,7 & 4,7 & 13,5 & 1,7 & 9,8 & 18,2 & 11,1 \\
\hline 2011 & 75,3 & 64,2 & 4,7 & 13,8 & 1,7 & 10,2 & 18,2 & 11,8 \\
\hline 2012 & 74,5 & 61,4 & 4,8 & 13,9 & 2,1 & 11,8 & 18,6 & 12,9 \\
\hline 2013 & 73,7 & 59,6 & 4,6 & 12,1 & 2,6 & 14,1 & 19,1 & 14,2 \\
\hline
\end{tabular}

Fuente: Afiliación de trabajadores al Sistema de la Seguridad Social, Estadísticas del Ministerio de Empleo y Seguridad Social.

Los empresarios agrícolas han sabido poner este marco institucional discriminatorio al servicio de la competitividad de la agroindustria, desarrollando diversas estrategias orientadas a asegurarse la disposición de la cantidad necesaria de trabajadores, con la cualificación adecuada y con una actitud no conflictiva, durante todo el tiempo de la campaña y a un coste lo más bajo posible. En el logro de este objetivo han jugado un papel fundamental las estrategias empresariales de sustitución étnica y la externalización del reclutamiento de la mano de obra, mediante la utilización de intermediarios informales (Castellanos y Pedreño, 2001), programas de contratación en origen (Gualda y Ruíz, 2005; Moreno, 2009; Gordo y Felicidades, 2009; Reigada, 2011; Gualda, 2012) o empresas de trabajo temporal (Andreo et al., 2005; Andreo, 2007;). La agricultura ha constituido, además, la puerta de entrada al mercado laboral para una buena parte de trabajadores inmigrantes en situación irregular 
que, con el tiempo, regularizaban su situación mediante procedimientos de arraigo. Una vez obtenido el permiso de trabajo y residencia, una parte de estos migrantes abandonaban la agricultura en busca de mejores ocupaciones en la construcción y los servicios. Este proceso ha constituido el itinerario laboral tipo de muchos trabajadores inmigrantes, pero también ha funcionado como mecanismo de sostenimiento de la economía sumergida y de reproducción de un ejército de reserva altamente funcional a las estrategias empresariales de sustitución étnica.

La agricultura salarial se ha constituido, de este modo, como un espacio ocupacional de y para trabajadores y trabajadoras extranjeros. En 2007, el año de mayor afiliación, los trabajadores extranjeros suponían el 8,9\% del total de afiliados y el 13,4\% de los afiliados en el sistema especial agrario. Esta sobrerrepresentación ha crecido con la crisis económica lo que refleja la permanencia de estos trabajadores en la agricultura, aunque en los dos últimos años su peso haya disminuido ligeramente. En 2013, los trabajadores extranjeros representan el 9,2\% del total de afiliados y el 22,5\% en el Sistema Especial Agrario (tabla 2).

TABLA 2

AFILIADOS A LA SEGURIDAD SOCIAL SEGÚN NACIONALIDAD, TOTAL DE REGÍMENES Y SISTEMA ESPECIAL AGRARIO, 2005-2013 (\% SOBRE EL TOTAL DE AFILIADOS)

\begin{tabular}{|c|c|c|c|c|c|c|}
\cline { 2 - 7 } \multicolumn{1}{c|}{} & \multicolumn{3}{c|}{ Total regímenes } & \multicolumn{3}{c|}{ Sistema Especial Agrario } \\
\cline { 2 - 7 } \multicolumn{1}{c|}{} & Españoles & Extranjeros & \% Ext. & Españoles & Extranjeros & $\%$ Ext. \\
\hline 2005 & 17.835 .400 & 1.461 .140 & 5,8 & 1.043 .700 & 143.058 & 9,7 \\
\hline 2006 & 18.596 .300 & 1.822 .406 & 7,6 & 999.300 & 154.060 & 12,1 \\
\hline 2007 & 19.152 .300 & 1.975 .578 & 8,9 & 968.900 & 150.353 & 13,4 \\
\hline 2008 & 19.005 .600 & 2.052 .406 & 9,4 & 744.500 & 183.762 & 13,4 \\
\hline 2009 & 17.916 .800 & 1.878 .023 & 9,7 & 802.200 & 233.930 & 19,8 \\
\hline 2010 & 17.581 .900 & 1.840 .827 & 9,5 & 818.800 & 248.698 & 22,6 \\
\hline 2011 & 17.326 .300 & 1.783 .858 & 9,5 & 820.200 & 246.140 & 23,3 \\
\hline 2012 & 16.738 .600 & 1.693 .324 & 9,3 & 810.100 & 234.955 & 23,1 \\
\hline 2013 & 16.227 .652 & 1.588 .661 & 9,2 & 748.290 & 192.387 & 22,5 \\
\hline
\end{tabular}

Fuente: Afiliación de trabajadores al Sistema de la Seguridad Social, Estadísticas del Ministerio de Empleo y Seguridad Social. 
El mapa 1 muestra la importancia de la agricultura como nicho laboral para los trabajadores inmigrantes, especialmente en provincias como Huelva, Almería, Murcia o Albacete, donde la concentración de los extranjeros en el sistema especial agrario supera el $40 \%$ del total. También los diferentes estudios de caso revelan esta tendencia a la sobreconcentración del colectivo inmigrante en las tareas de jornalerismo agrícola, especialmente en el caso de los marroquíes y ecuatorianos (Barbolla, 2001; Checa, 1995; Pedreño, 1999). Los colectivos rumanos y búlgaros también tienen una presencia significativa en el sector agrícola en las agriculturas de ciertas zonas geográficas (Viruela, 2003 y 2006). Esta etnificación también se refleja en el hecho de que son los trabajadores inmigrantes extranjeros los que ocupan las ocupaciones menos cualificadas, con peores condiciones salariales y menor prestigio.

Pero la segmentación del trabajo agrícola no se agota en la cuestión étnica. Estamos ante mercados laborales donde el género constituye una variable fundamental para entender su funcionamiento. De un lado, cabe señalar que la importante presencia de mujeres en determinadas producciones o actividades agrícolas no es un fenómeno nuevo. Desde los inicios de la agricultura intensiva en España, las mujeres han sido la fuerza de trabajo mayoritaria en las tareas de manipulado del producto agrícola en las conserveras y en los almacenes, tareas feminizadas a las que se incorporaron las mujeres inmigrantes a su llegada (Pedreño, Gadea y de Castro, 2014). 


\section{MAPA 1}

PORCENTAJE DE TRABAJADORES EXTRANJEROS AFILIADOS A LA SEGURIDAD SOCIAL EN ALTA LABORAL EN EL SISTEMA ESPECIAL AGRARIO, 2013 (\% SOBRE EL TOTAL DE TRABAJADORES EXTRANJEROS EN ALTA LABORAL).

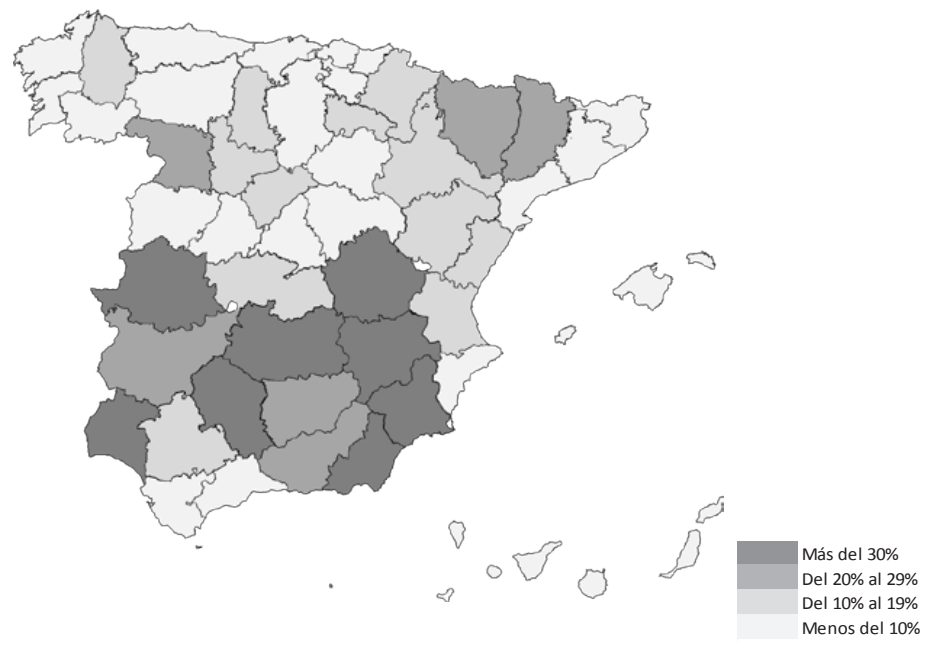

Elaboración propia a partir de datos de Afiliación de trabajadores al Sistema de la Seguridad Social, Estadísticas del Ministerio de Empleo y Seguridad Social.

De otro, determinados procesos de sustitución étnica en los campos han llevado a la configuración de mercados laborales masculinizados o feminizados. Es el caso del cultivo de la fresa en Huelva, donde a través de los programas de contratación en origen la fuerza de trabajo local fue parcialmente sustituida, primero, por varones procedentes de Marruecos y, con posterioridad, por mujeres de Europa del Este y de África (Gualda y Ruíz, 2005; Gualda, 2012; Reigada, 2011 y 2013).

\subsection{El impacto de la crisis en el trabajo agrícola}

La concentración en determinados sectores y ocupaciones ha situado a los trabajadores inmigrantes en una posición de vulnerabilidad que se ha visto rápidamente degradada con la crisis económica. En 2006, 
antes de que la actual crisis económica se materializara en una destrucción de empleo sin precedentes, la tasa de paro se situaba en el 7,9\% para los trabajadores españoles y un 11,7\% para los extranjeros (el 12,1\% para aquellos no pertenecientes a la Unión Europea). En 2014, la tasa de paro de los españoles se sitúa en el $23 \%$ mientras que la de los extranjeros alcanza el 34,5\% y llega hasta el 37,4\% en el caso de los no comunitarios. Los datos de evolución de la ocupación desde el inicio de la crisis muestran el tremendo ritmo de destrucción del empleo en el mercado laboral español: entre 2008 y 2014 el número de ocupados ha descendido en 3.125.500 personas, de las que 1.466 .400 pertenecen al sector de la construcción (un 59\% de ocupados menos). Respecto a la agricultura, las cifras muestran que entre 2008 y 2014 se ha producido una pérdida de 92.400 ocupados $(-11,16 \%)$, de los que 47.100 fueron varones $(-7,78 \%)$ y 45.300 mujeres $(-20,34 \%)$. Si analizamos los datos por nacionalidad, encontramos que la ocupación de españoles y extranjeros ha seguido una tendencia diferente: los trabajadores españoles descienden entre 2008 y 2012 y crecen en los dos últimos años en 19.600 ocupados; por el contrario, los trabajadores extranjeros aumentan hasta 2012 para disminuir los dos últimos años un 16,2\%, lo que representa 26.800 ocupados menos (tabla 3 ).

\section{TABLA 3}

OCUPADOS EN EL SECTOR DE LA AGRICULTURA POR NACIONALIDAD Y SEXO, ESPAÑA 2008 Y 2014 (EN MILES)

\begin{tabular}{|l|r|r|r|r|r|r|r|r|r|}
\cline { 2 - 10 } \multicolumn{1}{c|}{} & \multicolumn{3}{c|}{ Total } & \multicolumn{3}{c|}{ Española } & \multicolumn{3}{c|}{ Extranjera } \\
\cline { 2 - 11 } & $\begin{array}{c}\text { Ambos } \\
\text { sexos }\end{array}$ & Hombres & Mujeres & $\begin{array}{c}\text { Ambos } \\
\text { sexos }\end{array}$ & Hombres & Mujeres & $\begin{array}{c}\text { Ambos } \\
\text { sexos }\end{array}$ & Hombres & Mujeres \\
\hline 2008 & 828,2 & 605,5 & 222,7 & 672,8 & 488,5 & 184,3 & 152,8 & 115,5 & 37,3 \\
\hline 2009 & 788,1 & 582,9 & 205,2 & 628,8 & 461,3 & 167,6 & 155,7 & 119,8 & 35,9 \\
\hline 2010 & 786,1 & 583,1 & 203 & 622,8 & 455,4 & 167,4 & 157,9 & 124,6 & 33,3 \\
\hline 2011 & 755,3 & 557,5 & 197,8 & 589,8 & 430,1 & 159,7 & 157,2 & 121,8 & 35,3 \\
\hline 2012 & 743,4 & 549,6 & 193,8 & 570,3 & 419,4 & 150,9 & 165,2 & 124,0 & 41,3 \\
\hline 2013 & 736,6 & 561,1 & 175,4 & 579,7 & 438,1 & 141,6 & 151,4 & 118,1 & 33,3 \\
\hline 2014 & 735,8 & 558,4 & 177,4 & 589,9 & 448,9 & 141 & 138,4 & 102,9 & 35,5 \\
\hline Var. Abs. & $-92,4$ & $-47,1$ & $-45,3$ & $-82,9$ & $-39,6$ & $-43,3$ & $-14,4$ & $-12,6$ & $-1,8$ \\
\hline$\%$ Var. & $-11,16$ & $-7,78$ & $-20,34$ & $-12,32$ & $-8,11$ & $-23,49$ & $-9,42$ & $-10,91$ & $-4,83$ \\
\hline
\end{tabular}

Fuente: Encuesta de Población Activa, INE. 
Entre los trabajadores de nacionalidad española, aunque los varones pierden un total de 39.600 efectivos (el 8,11\%), se registra un incremento en los dos últimos años: en 2013 y 2014 han sumado 29.500 ocupados. Las ocupadas españolas, por su parte, no han dejado de disminuir desde 2008, con una pérdida total de 43.300 personas (el 23,5\%). En el caso de los trabajadores extranjeros, los varones registran una pérdida total de 12.600 ocupados entre 2008 y 2014, pero lo más relevante es que en los dos últimos años esa pérdida ha sido de 21.100 personas. Entre las mujeres extranjeras la pérdida de ocupados ha sido menor, un total de 1.800 ocupadas $(-4,83 \%)$.

En los primeros momentos de la crisis se habría producido una vuelta a la agricultura de trabajadores extranjeros, principalmente varones que habían perdido su empleo en la construcción, la industria y los servicios (Pajares, 2010). Sin embargo, la capacidad de absorción de este sector es limitada y, recientemente, se ha producido un descenso en la contratación de trabajadores extranjeros (Viruela y Torres, 2015). Respecto a los trabajadores españoles, su retorno a la agricultura no se habría producido en los primeros momentos de la crisis, sino en los últimos años. Aunque los datos no nos permiten afirmarlo con rotundidad, podríamos apuntar aquí que esta vuelta se produce cuando previsiblemente muchos de estos trabajadores han visto fuertemente deteriorada su situación económica, por el agotamiento de ahorros y prestaciones. De este modo, gracias a la crisis económica, la agricultura salarial cuenta de nuevo con un ejército de mano de obra disponible, vulnerable y altamente disciplinado.

\section{JORNALEROS INMIGRANTES EN LA AGRICULTURA INTENSIVA MURCIANA}

Murcia es uno de los principales enclaves de agricultura intensiva que han surgido en el Sur de Europa en el contexto de la globalización del sistema agroalimentario. Desde finales de los años 80, la estrategia de desarrollo de la región ha consistido en insertarse en las redes de la economía global por medio de la producción intensiva de frutas y hortalizas para la exportación en fresco (Pedreño, 2005). 
$\mathrm{Al}$ igual que en otras regiones españolas, en Murcia el desarrollo de la agroindustria y la mayor demanda de trabajo asalariado coincidieron con el abandono de la agricultura por parte de los jornaleros autóctonos. De igual manera, la respuesta empresarial a la necesidad de mano de obra abundante y barata se ha basado en la recreación de las relaciones jornaleras tradicionales y la movilización de categorías sociales vulnerables, con mujeres e inmigrantes como principales protagonistas (Pedreño, 1998; Segura y Pedreño, 2006), lo que ha dado lugar a un mercado de trabajo segmentado por género y etnia.

Los primeros inmigrantes comienzan a llegar a los municipios agrícolas de Murcia a finales de los años 80, varones jóvenes procedentes del Magreb, que se van instalando en la zona siguiendo las necesidades de la agricultura intensiva. Este perfil de la inmigración se mantendrá hasta finales de los años 90, cuando comienza a modificarse con la diversificación de nacionalidades, la mayor presencia de mujeres y los procesos de reagrupación familiar y formación de nuevas familias (Torres, 2007). Los patrones de movilidad de los jornaleros también se han transformado. En los años 80 y 90 encontrábamos migraciones de tipo circular y pendular, ya que la región constituía un punto importante en los desplazamientos de los jornaleros a lo largo de todo el territorio español siguiendo las diferentes cosechas; también eran frecuentes las migraciones pendulares, sobre todo de marroquíes que retornaban a sus lugares de origen en los momentos de menor trabajo o de paro en la agricultura. En la actualidad, el patrón predominante es el asentamiento en los municipios murcianos, ya que los procesos de intensificación de la agricultura permiten a estos jornaleros trabajar durante casi todo el año (Gadea, Ramírez y Sánchez, 2014).

La agroindustria murciana se ha ido configurando, desde los años noventa como un nicho de trabajo inmigrante. Los primeros extranjeros, en su mayoría varones, sustituyeron a los antiguos jornaleros en los campos, mientras que en los almacenes se mantenían las trabajadoras autóctonas. Se reforzaba así una segmentación por género que ha caracterizado a la agroindustria murciana desde sus inicios. Con la llegada de la migración ecuatoriana, con una sex ratio más equilibrada, la presencia de mujeres inmigrantes en los campos y almacenes comenzó a ser cada vez mayor, y la posición subordinada de la mujer en el trabajo agrícola se transfirió a las nuevas trabajadoras de origen inmigrante. 
En 2001, los trabajadores extranjeros ya representaban el 43,4\% del total de trabajadores del Sistema Especial Agrario (tabla 4). Su peso fue creciendo a la largo de la década, hasta alcanzar el 72,4\% en 2008, con un total de 41.423 afiliados extranjeros frente a 16.141 españoles. En los tres últimos años, los trabajadores extranjeros afiliados en el Sistema Especial han descendido en 10.423 efectivos $(-24,2 \%)$, mientras los españoles han aumentado en 9.950 (un $46,3 \%$ ). Los datos muestran claramente un retorno significativo de trabajadores españoles a la agricultura, con una presencia similar a la de hace una década.

TABLA 4

TRABAJADORES AFILIADOS EN EL SISTEMA ESPECIAL AGRARIO POR NACIONALIDAD, REGIÓN DE MURCIA, 2001-2013

\begin{tabular}{|l|c|c|c|}
\cline { 2 - 4 } \multicolumn{1}{c|}{} & Españoles & Extranjeros & \% Extranjeros \\
\hline 2001 & 30.118 & 23.082 & 43,4 \\
\hline 2002 & 26.428 & 32.572 & 55,2 \\
\hline 2003 & 33.309 & 32.791 & 49,6 \\
\hline 2004 & 29.631 & 29.269 & 49,7 \\
\hline 2005 & 18.318 & 41.423 & 69,3 \\
\hline 2006 & 23.301 & 37.936 & 61,9 \\
\hline 2007 & 19.404 & 36.466 & 65,3 \\
\hline 2008 & 16.141 & 42.359 & 72,4 \\
\hline 2009 & 20.789 & 44.211 & 68,0 \\
\hline 2010 & 20.616 & 44.012 & 68,1 \\
\hline 2011 & 21.488 & 43.107 & 66,7 \\
\hline 2012 & 23.262 & 42.546 & 64,7 \\
\hline 2013 & 31.438 & 32.684 & 51,0 \\
\hline
\end{tabular}

Fuente: Afiliación de trabajadores al Sistema de la Seguridad Social, Estadísticas del Ministerio de Empleo y Seguridad Social.

Respecto a las condiciones laborales en el sector, el mercado de trabajo agrícola murciano se ha conformado a partir de una norma 
de empleo caracterizada por la informalidad, la eventualidad, la intensificación y flexibilización del tiempo de trabajo, el alargamiento de las jornadas y los bajos salarios. Esta norma de empleo agrícola, que debilita los vínculos entre trabajo y ciudadanía, tiene importantes repercusiones en las condiciones de vida y trabajo de los jornaleros (de Castro, 2014). Las continuas entradas y salidas del mercado laboral se traducen en vidas marcadas por la temporalidad, la movilidad sectorial y territorial, y la combinación de actividades formales e informales.

Los últimos datos publicados por el Servicio Estatal de Empleo sobre el mercado de trabajo de los extranjeros en la Región de Murcia muestran que, en el año 2011, se realizaron 249.345 contratos a extranjeros (un $43,76 \%$ del total del contratos), concentrados fundamentalmente en dos actividades: «actividades relacionadas con el empleo ${ }^{6}(142.351$ contratos, el 57\%) y «agricultura, ganadería, caza y actividades de los servicios relacionados con las mismas» (59.973 contratos, el 24\%). El 92,7\% de estos contratos se registraron en la modalidad de «obra y servicio» $\mathrm{y}$ «eventual por circunstancias de la producción», lo que da cuenta de la fuerte temporalidad en la contratación, a lo que habría que unir la elevada la rotación ${ }^{7}$, con un índice de 3,2 contratos por persona (SEPE, 2012). Estos datos nos permiten aproximarnos a los principales rasgos del mercado de trabajo de los extranjeros: concentración en actividades agrarias, temporalidad, alta rotación e importancia de la contratación a través de Empresas de Trabajo Temporal. Precisamente, este tipo de empresas habría jugado un papel fundamental tanto en la etnificación del trabajo agrícola como en la elevada temporalidad y rotación de la contratación a trabajadores extranjeros, un fenómeno que ya destacaban algunos autores a mediados de la década pasada (Andreo et al., 2005; Andreo, 2007) y que en el contexto de crisis se ha

6 Esta categoría incluye los contratos realizados por Empresas de Trabajo Temporal y Agencias de Colocación, que ceden trabajadores a otras empresas, fundamentalmente del sector agrícola.

$7 \quad$ El índice de rotación relaciona el número de contratos con el número de personas contratadas en el año. La Región de Murcia ha registrado 249.345 contratos de este colectivo que han dado empleo a 77.432 personas, siendo su índice de rotación de 3,22 c/p. Esto se traduce en que, por término medio, a un mismo trabajador le han realizado más de tres contratos al año (SEPE, 2012). 
intensificado. Entre 2005 y 2013, los contratos de puesta a disposición $^{8}$ han aumentado en un 165\% (de 160.882 a 265.567 contratos). En el caso del sector agrario, este incremento ha sido todavía mayor: de 105.398 contratos en 2005 a 229.871 en 2013, lo que supone un aumento del $218 \%$.

La precariedad en las condiciones de trabajo se ha traducido en una vulnerabilidad en las condiciones de vida. A pesar de ello, antes de la actual crisis económica, una parte importante de los inmigrantes pioneros habían conseguido una estabilidad laboral y residencial que les permitió llevar a cabo procesos de reagrupación familiar y formación de nuevas familias. Otra parte de la inmigración estaba aún en el momento de llegada, con situaciones diversas, ya que junto a personas y familias que no habían conseguido regularizar y estabilizar su situación, se encontraban aquellos que habían llegado recientemente (Torres, 2007). La crisis económica ha supuesto un punto de inflexión en los procesos de inserción de los vecinos inmigrantes.

La destrucción de empleo ha sido mayor en este colectivo, especialmente al inicio de la crisis, donde las tasas de paro de los extranjeros llegaron a duplicar a las de los españoles: un 33,94\% frente al $16,6 \%$ respectivamente en 2009 , alcanzando el $40,1 \%$ en el caso de los varones extranjeros. Como muestran diversos autores, esta mayor incidencia responde a la concentración de los trabajadores inmigrantes en los sectores más golpeados por la crisis y en las categorías laborales de menor cualificación y su mayor índice de temporalidad (Pajares, 2010; Mahía y Del Arce, 2010). Con posterioridad, la destrucción de empleo en todos los sectores ha hecho que estas diferencias se reduzcan, aunque en 2013 la tasa de paro de los extranjeros $(37,6 \%)$ todavía era superior en diez puntos a la de los españoles $(27,1 \%)$.

Junto al desempleo, los trabajadores tienen que enfrentarse a una degradación de las condiciones laborales. En el actual contexto de crisis y agrandamiento del ejército de reserva, las empresas del sector están acentuando sus estrategias de competitividad basadas

8 En la Región de Murcia, el sector agrario concentra la mayoría de contratos de puesta a disposición (contratos mercantiles que establecen las Empresas de Trabajo Temporal con las empresas usuarias para la cesión de trabajadores), hasta el punto de que en 2013 el $86,6 \%$ de los contratos realizados pertenecen a este sector. 
en la reducción de costes laborales. Como muestran algunas investigaciones realizadas (Torres y Gadea, 2010 y 2012) y en curso, estamos asistiendo a una proliferación de las irregularidades laborales (destajos, ausencia de contrato, no remuneración de las horas extraordinarias, prestamistas informales de mano de obra, etc.), a una intensificación de los ritmos de trabajo y a una generalización de la precariedad laboral en un sector donde siempre persistió la eventualidad como relación contractual básica.

Además, la actual situación económica y las políticas de ajuste están desestabilizando dos de las condiciones que habían facilitado una inserción tranquila de los inmigrantes en el pasado más reciente: el trabajo y los servicios públicos. Al igual que ha mostrado Avallone (2014) para el caso italiano, la presencia de trabajadores migrantes en la Región de Murcia había favorecido los procesos de movilidad social ascendente de una parte de la población rural, que abandonó la agricultura para ocupar empleos con mayor cualificación y mejores condiciones laborales. Con la crisis, el carácter complementario de los trabajadores autóctonos e inmigrantes que había caracterizado la estructura productiva etnofragmentada se pone en cuestión, con una cierta vuelta de trabajadores españoles al campo y el incremento de las situaciones de competencia. Por otro lado, la reducción del gasto público limita las coberturas y facilita el surgimiento de tensiones entre vecinos precarios de unos y otros orígenes (Torres y Gadea, 2012).

\section{CONCLUSIONES: SOBRE LA ESPECIFICIDAD DE LA CRISIS EN LAS ZONAS AGRÍCOLAS}

El desarrollo de la agricultura intensiva de exportación genera importantes beneficios económicos y ha permitido crear una gran cantidad de puestos de trabajo en diversas regiones españolas. Se trata, sin embargo, de un crecimiento económico que se ha realizado a costa de la precariedad de las condiciones de vida y trabajo de los jornaleros agrícolas, buena parte de los cuales son trabajadores inmigrantes. Para garantizar la competitividad de los productos agrícolas en los mercados globales, las empresas del sector, con el apoyo de las instituciones públicas, han promovido la implantación de una norma de empleo basada en la informalidad, la 
elevada estacionalidad, la intensificación y flexibilización del tiempo de trabajo, el alargamiento de las jornadas y los bajos salarios. En efecto, la agricultura intensiva ha basado su competitividad en la contención de los costes laborales y en una gestión flexible del trabajo.

La figura y la posición del trabajador eventual cuenta con una larga tradición, no sólo en la Región de Murcia, sino también en el conjunto del Sur de Europa. Los efectos de esta eventualidad sobre los trabajadores se traducen en insuficiencia salarial, inestabilidad y precariedad vital. De hecho, podríamos afirmar que la agricultura intensiva depende de la existencia de una población socio-económicamente vulnerable, renovada y reproducida de manera permanente, que permanezca en los campos para responder, rápida y disciplinadamente, a las necesidades de una agricultura hiperflexible, que produce importantes beneficios en los eslabones más altos de la cadena gracias a la degradación de las condiciones laborales, salariales y, por tanto, vitales de los trabajadores.

Los trabajadores inmigrantes han jugado un papel fundamental en estas estrategias, ocupando la posición de los trabajadores autóctonos dentro del ejército de reserva en los campos. Su vulnerabilidad, derivada en gran parte de los mecanismos políticos y empresariales que lo han construido como trabajador y complemento del autóctono, los ha condenado a insertarse en sectores precarizados y frágiles desde el punto de vista de los derechos laborales. En la actual situación de crisis, con elevadas tasas de desempleo y fuertes recortes sociales, su frágil posición en la estructura laboral y social no ha tardado en degradarse. A esto habría que añadir un cambio en el principio de complementariedad que había regido la posición a inmigrantes y autóctonos en el mercado de trabajo agrícola. Si en los primeros momentos de la crisis económica se constataba una vuelta a la agricultura de trabajadores extranjeros que habían perdido su empleo en la construcción, la industria y los servicios, en los últimos años se observa un retorno de trabajadores autóctonos, presumiblemente en un contexto de fuerte deterioro de su situación económica, por el agotamiento de ahorros y prestaciones. De este modo, gracias a la crisis económica, la agricultura cuenta de nuevo con un ejército de mano de obra disponible, vulnerable y altamente disciplinado. 


\section{BIBLIOGRAFÍA}

Achón, O. (2011): Importando miseria. La alternativa a la provisión de mano de obra agrícola, Madrid, Catarata

Andreo, J.C.; Guerrero, Ma J.; Arcos, B. y GÁlvez, D. (2005): «Intermediación en el mercado laboral de mano de obra inmigrante extranjera en la Región de Murcia: el caso de las empresas de trabajo temporal», Papeles de geografía, 41-42, pp. 51-70

AndReO, J.C. (2007): Inmigración extranjera y empresas de trabajo temporal en la Región de Murcia, Madrid, Doble J, Colección Ciencias Sociales.

Avallone, G. (2014): «El campo neoliberal y su crisis. Agricultura, sociedad local y migraciones en la Europa del Sur», Encrucijadas, 6, pp. 39-55

Avellá, L. y García, M. (1995): «La inmigración en las agriculturas mediterráneas», Revista Española de Economía Agraria, 172, pp. 51-76.

Barbolla, D. (2001): Inmigración marroquí en la zona de Talayuela (Cáceres) 1992-1996, Mérida, Editora Regional de Extremadura.

Castellanos, M ${ }^{\mathrm{a}}$ L. y Pedreño, A. (2001): «Desde el Ejido al accidente de Lorca. Las amargas cosechas de los trabajadores inmigrantes en los milagrosos vergeles de la agricultura mediterránea», Sociología del Trabajo, 42, pp. 3-31.

Castro, C. de (2014): «La desdemocratización de las relaciones laborales en los enclaves globales de producción agrícola», en A. Pedreño (coord.), De cadenas, migrantes y jornaleros. Los territorios rurales en las cadenas globales agroalimentarias. Madrid, Talasa, pp. 59-77.

Castro, C. de; Gadea, E. y Pedreño, A. (2014): «Inmigración, crisis del Sur de Europa y y sostenibilidad social de las estrategias de desarrollo. El caso de los enclaves productivos de agricultura intensiva», Revista Trabajo, 11 , pp. 87-111.

CHecA, F. (1995): «Oportunidades socioeconómicas en el proceso migratorio de los inmigrantes africanos en Almería», Agricultura y Sociedad, 77, pp. 41-82.

Doeringer, P. y Piore, M. (1985): Mercados internos de trabajo y análisis laboral, Madrid, Ministerio de Trabajo y Seguridad Social. [1971]

GadeA, E; Ramírez, A y SÁnchez, J. (2014): «Estrategias de reproducción social y circulaciones migratorias de los trabajadores en los enclaves globales», en A. Pedreño (coord.), De cadenas, migrantes y jornaleros. Los territorios rurales en las cadenas globales agroalimentarias. Madrid, Talasa, pp. 134-149.

GiméNEZ, C. (1992): «Trabajadores extranjeros en la agricultura española, enclaves e implicaciones», Estudios Regionales, 31, pp. 121-147. 
Gordo, M. y Felicidades, J. (eds.), (2009): Explorando los contratos en origen en los campos españoles, Huelva, Servicio de Publicaciones de la Universidad de Huelva.

GozÁlvez, V. y López, L. (1999): «Jornaleros extranjeros en el campo español», Ería. Revista cuatrimestral de geografía, 49, pp. 213-230.

Gualda, E. y Ruíz, M. (2005): «Procesos de sustitución étnica e inserción sociolaboral de mujeres extranjeras en Huelva», Trabajo: Revista andaluza de relaciones laborales, 15 , pp. 177-193

Gualda, E. (2012): «Migración circular en tiempos de crisis. Mujeres de Europa del Este y africanas en la agricultura de Huelva», Papers, 97 (3), pp. 613-640.

Leborgne, D. y Lipietz, A. (1994): «Flexibilidad ofensiva, flexibilidad defensiva. Dos estrategias sociales en la producción de los nuevos espacios económicos», en G. Benko y A. Lipietz (eds.), Las Regiones que Ganan. València, Edicions Alfons el Magnànim.

Magrama (2014): Informe Anual de Comercio Exterior, 2013. Madrid, Ministerio de Agricultura, Alimentación y Medio Ambiente.

MAHÍA, R. y DEL ARCE, R. (2010): Impacto de la crisis laboral sobre la población inmigrante. Madrid, Real Instituto Elcano.

Moreno, J. (2009): "Los contratos en origen de temporada: mujeres marroquíes en la agricultura onubense», Revista de Estudios Internacionales Mediterráneos, 7, pp. 58-78

Pajares, M. (2010): Inmigración y mercado de trabajo. Informe 2010. Madrid, Observatorio Permanente de la Inmigración.

Pedreño, A. (1998): Del jornalero agrícola al obrero de las factorías vegetales. Madrid, Ministerio de Agricultura, Pesca y Alimentación.

Pedreño, A. (1999): «Taylor y Ford en los campos: trabajo, género y etnia en el cambio tecnológico y organizacional de la agricultura industrial murciana», Sociología del Trabajo, 35, pp. 25-56.

Pedreño, A. (2005): «Sociedades etnofragmentadas», en A. Pedreño y M. HERNÁNDEZ (coords.), La condición inmigrante. Exploraciones e investigaciones desde la Región de Murcia. Murcia, Ediciones de la Universidad de Murcia, pp. 75-106

Pedreño, A.; Gadea, E. y Castro, C. de (2014): «Labor, Gender, and Political Conflicts in the Global Agri-food System: The Case of the Agri-export Model in Murcia, Spain», en A. Bonanno y J. Cavalcanti (eds.) Labor Relations in Globalized Food (Research in Rural Sociology and Development, Volume 20), Emerald Group Publishing Limited, pp. 193-214 
Reigada, A. (2011): "Más allá del discurso sobre la 'inmigración ordenada': contratación en origen y feminización del trabajo en los campos de fresas», Politica y Sociedad, 49, 1, pp. 103-122.

Reigada, A. (2013): «Feminización de la inmigración y el trabajo en la agricultura de exportación: el caso del monocultivo de la fresa en Andalucía», en SÁnchez, M. J. y SERra, I. (coords.), Ellas se van. Mujeres migrantes en Estados Unidos y España, México, Instituto de Investigaciones Sociales de la UNAM, pp. $199-234$.

Segura, P. y Pedreño, A. (2006): «La hortofrutícultura intensiva de la Región de Murcia: un modelo productivo diferenciado», en: M. EtXezarReta, La agricultura española en la era de la globalización. Madrid, Ministerio de Agricultura, Pesca y Ganadería, pp. 369-421.

SEPE (2012): Informe del mercado de trabajo de los extranjeros, Murcia. Datos 2011. Madrid, Ministerio de Empleo y Seguridad Social.

SEPE (2013): Estudio Prospectivo. El Sector Hortofrutícola en España 2012. Madrid, Ministerio de Empleo y Seguridad Social.

Torres, F. (dir.) (2007): Los nuevos vecinos de la mancomunidad del sureste, los inmigrantes y su inserción en Torre Pacheco, Fuente Álamo y La Unión (Murcia). Murcia, Universidad de Murcia.

Torres, F. y GADEA, E. (2010): «Inserción laboral de los inmigrantes, estructura etno-fragmentada y crisis económica. El caso del Campo de Cartagena (Murcia)», Sociología del Trabajo, 69, pp. 73-94

Torres, F. y Gadea, E. (2012): "Agricultura intensiva de exportación, inmigración y transformación rural. El caso del Campo de Cartagena, 19902010 (Murcia)», comunicación presentada al IX Coloquio Ibérico de Estudios Rurales, Lisboa, 27 y 28 de julio de 2012.

Torres, T., Allepuz, R., Gordo, M. (2014): «La contratación de mano de obra temporal en la agricultura hortofrutícola española», Ager. Revista de Estudios sobre Despoblación y Desarrollo Rural, 16, pp. 7-37.

Viruela, R. (2003): "La nueva corriente inmigratoria de Europa del Este», Cuadernos de Geografía, 72, pp. 231-258.

VIRUELA, R. (2006): «Inmigrantes rumanos en España: aspectos territoriales y procesos de sustitución laboral», Scripta Nova, Revista Electrónica de Geografía y Ciencias Sociales, X, 222.

Viruela, R, y Torres, F. (2015): «Flujos migratorios, crisis y estrategias de movilidad. Los inmigrantes ecuatorianos y rumanos en España», en ToRRES, F. y GADEA, M M $^{\mathrm{a}}$. (coords.), Crisis, inmigración y sociedad. Madrid, Talasa, pp. 37-72. 